\title{
Facial Expression Recognition for Color Images using Gabor, Log Gabor Filters and PCA
}

\author{
Shail Kumari Shah \\ PG Scholar, \\ Computer Engg. Dept. \\ Rajasthan College of Engineering for Women \\ Rajasthan Technical University, Jaipur, India
}

\author{
Vineet Khanna \\ Assistant Professor, \\ Computer Engg. Dept. \\ Rajasthan College of Engineering for Women \\ Rajasthan Technical University, Jaipur, India
}

\begin{abstract}
Facial expression recognition is an interesting and challenging problem, and found in many applications like humancomputer interaction (HCI), robotics, video surveillance, border security, clinical research, person verification, crime prevention etc.. Facial expression is the movement of the muscles beneath the skin of the face. Through facial expressions human can convey their emotions without any verbal means. In this paper we have created raw database of color images. Training and testing set of images are created. Color information in an image is used to detect the face from the image. Important features from the detected face are extracted to form feature vectors using Gabor and Log Gabor filters. Principal Component Analysis (PCA) is used to reduce the dimension of the extracted features. Then these reduced features are classified using Euclidean distance. The main aim is to work upon three emotions-happy, neutral, surprise. Experiment carried out on self-generated database show comparable performance between Gabor and Log Gabor filters, where Log Gabor filters outperforming Gabor filters with classification accuracy of $86.7 \%$.
\end{abstract}

\section{Keywords}

Gabor filters, Log Gabor filters, PCA, Euclidean Distance, YCbCr, morphological, FER.

\section{INTRODUCTION}

Human beings can show their emotions through facial expressions. It is the movement of the muscles of the face. To detect these facial expressions is an interesting and tedious task. The research study by Mehrabian [9] has reported that 7 percent of the communication information is transferred by spoken words, $38 \%$ by voice intonation, and $55 \%$ by facial expressions of the speaker effects communication. Its applications are found in human computer intervention, AIBO robots, border security, medical science etc.. FACS (Facial Action Coding System) developed by Ekman and Friesen in 1978 describes the movement of different facial muscles which causes different facial expressions. Each component of facial movement is called $\mathrm{AU}_{\mathrm{s}}$ (Action Units).

There are two different approaches in Facial Expression Recognition (FER) system, geometric based and appearance based. Geometric based method is an old way. It consists of detecting and processing the movement of some facial points on image. The tracked spatial position and shapes of these facial points are used to form feature vectors for geometric based method. Rather than detecting and processing movements of these facial points, the parameters that vary within time, color (pixels), texture of related region are processed in appearance based method. In the presented approach various algorithms are used to recognize facial expressions. In the first algorithm input image is enhanced, intensity of the image is maintained, noise is removed. In the second algorithm Gabor and Log Gabor filers are used to form feature vectors. In third algorithm PCA is used for data reduction and in the next algorithm Euclidean distance is calculated to recognize accurate emotions.

\section{LITERATURE REVIEW}

On the past few decades there has been an increase in research on facial expression recognition due to its ever increasing applications in texture classification, facial analysis, human detection and many other tasks. Jyoti Mahajan et al. [1] proposed a method for an automatic FER system using artificial neural network. 18 feature values are extracted using 16 feature points from images to form feature vectors. These feature vectors were classified using feedforward back propagation algorithm. Jeemoni Kalita et al. [2] proposed Eigen vector based system. In this Eigen vectors and its Eigen values are computed. Mahesh Kumbhar et al. [3] used Gabor filters to extract features from the image. PCA is used for feature reduction and feed forward neural networks as classifier. In this system gray scale database called JAFFE database is used. Sayed Mehdi Lajevardi et al. [4] worked on color images and found that color components in color images provides more information than gray scale images to achieve improved and robust FER system. D.Murugan et al. [5] made comparison study on Gabor filters, Log Gabor filters and discrete wavelet transform. In their study it was found that Log Gabor filters works better for natural images compared to Gabor filters. There are various methods for feature reduction like LDA, PCA, and ICA. Saeid Fazli et al. [6] founded in their study that if the number of samples is less as compared to the dimensionality of the image then LDA alone is insufficient for feature reduction. To increase the performance PCA should be used before LDA. To reduce time complexity and dimension Hong-Bo Deng et al. [7] used PCA plus LDA. Maja Pantic et al. [8] gave some descriptions about FACS and requirements for selecting different database.

\section{SYSTEM ARCHITECTURE}

Here we propose facial expression recognition system by using various techniques. The system includes camera for capturing color images. Then the images are pre-processed to minimize the effect of environmental and other variations in an image. This includes scaling of an image, lightning compensation, noise removal, detection of eyes, mouth, nose to create mask of the face. Then the features are extracted and dimensions of an image are reduced in the second and third step. Lastly using the reduced features different emotions are classified. A block diagram description of FER system is shown in Fig 1. 


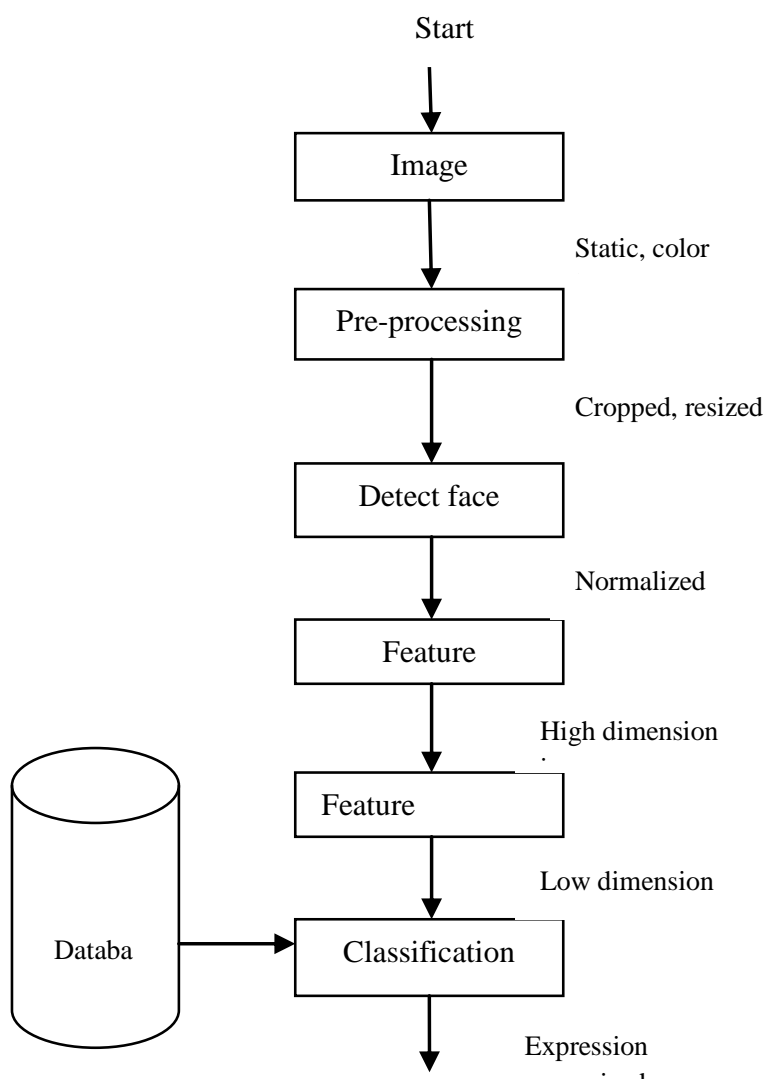

Fig 1: Block diagram of FER system

\section{IMAGE ACQUISITION}

Image is input to the system. Either static or dynamic image could be taken. Images could be 2-d monochrome (grayscale) or color images. However we have taken static, color image.

\section{IMAGE PRE-PROCESSING}

Image pre-processing is very important step in facial expression recognition task. In this step images are cropped and resized into same dimension. Finally the images are scaled into $180 \times 180$ pixels.

\section{FACE DETECTION}

Face detection may fail due to changes in shape, pose, lightning condition, occlusion, facial expressions. Therefore, to detect face correctly we have to go through the following steps.

\subsection{Lightning Compensation}

RGB color images are subjected to lightning condition and face detection may fail due to changes in lightning conditions. Therefore we normalize the image. Normalization is the process that changes and brings the pixel intensity values into the range that is most familiar or normal to the senses. It is also called contrast stretching. For this we have used "imadjust" function in MATLAB.

\subsection{Skin Extraction}

For skin extraction RGB images are converted into $\mathrm{YCbCr}$ color space. $\mathrm{Y}$ contains luminance information and $\mathrm{Cb}, \mathrm{Cr}$ contains chrominance information. Extracting skin reduces the time to search whole image for face detection. Skin color map of an image is derived from the chrominance component of the input image to detect pixels that are of skin color and the image is converted into binary image.

\subsection{Morphological Operations}

Morphological operations are performed to remove very small objects from the image while preserving the shape and size of larger objects in an image. Morphological opening of an image is detection of edge followed by dilation using structuring element. Detection of edge gives the end point of features like nose, eyes, mouth in an image. In the Dilation process fills the background pixels of an image. Some of the cavities are still left. Holes filling are done to fill the cavities that cannot be done using dilation.

\subsection{Bounding Box}

Bounding box is a fixed size window to detect final image. A quantified window size is selected to simply meet the size of the image.
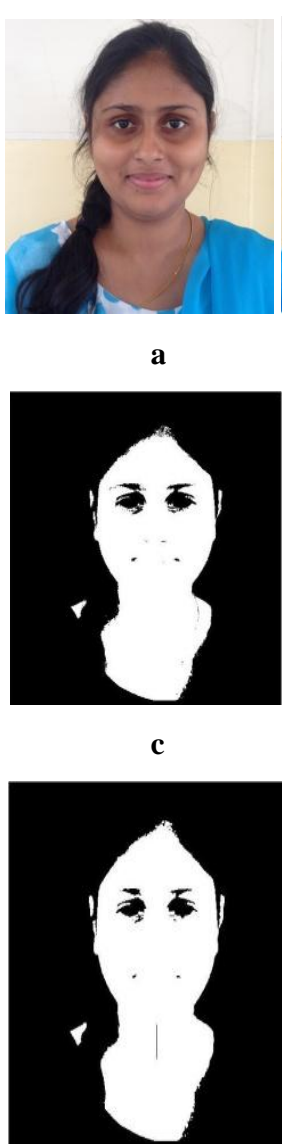

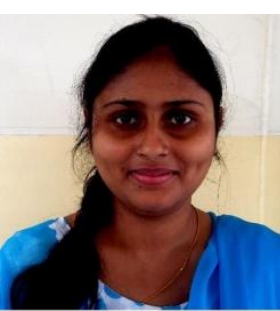

b
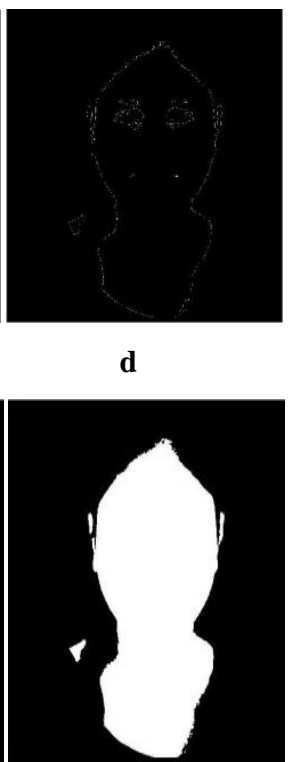

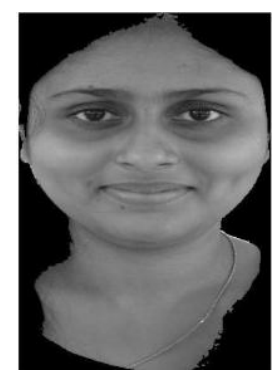

g

Fig 2: (a) original image (b) after lightning compensation (c) after skin extraction (d) after edge detection (e) after dilation (f) after holes filing (g) final face detection. 


\section{FEATURE EXTRACTION}

Feature extraction is a process of extracting essential information from large dataset. It is better to pass the normalized facial images to the feature extraction phase before it is given to the classifier. Feature extraction generally reduces the dimensionality of the input space while retaining essential information possessing high discrimination power and high stability []. We have used Gabor filter and Log Gabor filters in feature extraction phase.

\subsection{Gabor Filters}

Gabor filters are group of wavelets with each wavelet extracting features from the facial image aligned at certain angle and frequency. It is obtained by modulating amplitude of sinusoid with a Gaussian function.

A Gabor filters can be represented by the following equation:

$$
g(x, y)=s(x, y) w(x, y)
$$

Where $s(x, y)$ is a complex sinusoidal known as the carrier, and $w(x, y)$ is a 2-D Gaussian-shaped function known as the envelope. There are various forms to define this function, one normalized 2-d form being:

$$
\begin{aligned}
& g(x, y ; \lambda, \theta, \varphi, \sigma, \gamma) \\
& =\exp \left(-\frac{x^{\prime 2}+\gamma y^{\prime 2}}{2 \sigma^{2}}\right) \cos \left(2 \pi\left(2 \pi \frac{x^{\prime}}{\lambda}+\varphi\right)\right. \\
& g(x, y ; \lambda, \theta, \varphi, \sigma, \gamma) \\
& =\exp \left(-\frac{x^{\prime 2}+\gamma y^{\prime 2}}{2 \sigma^{2}}\right) \sin \left(i\left(2 \pi \frac{x^{\prime}}{\lambda}+\varphi\right)\right.
\end{aligned}
$$

Where

$$
x^{\prime}=x \cos \theta+y \sin \theta
$$

And

$$
y^{\prime}=-x \sin \theta+y \cos \theta
$$

The real and imaginary part of Gabor filter is shown in Fig 3.

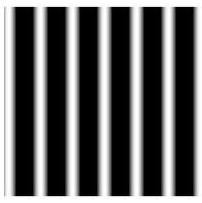

$\mathbf{a}$

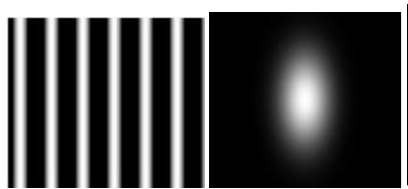

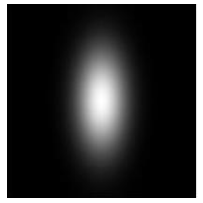

$\mathbf{b}$

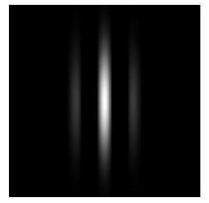

c

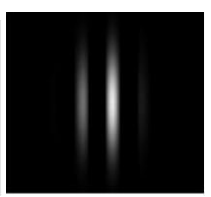

f
Fig 3: (a) real part of sinusoid (b) Gaussian envelop (c) real part of Gabor filter (d) imaginary part of sinusoid (e)

Gaussian envelop (f) imaginary part of Gabor filter

In this equation $(x, y)$ is the pixel position in spatial domain, $\lambda$ represents the wavelength (reciprocal of frequency) in pixels of sinusoidal plane wave, $\theta$ represents the orientation of the Gabor filter, $\psi$ is the phase offset, $\sigma$ is the spatial width of the Gaussian envelope and $\gamma$ is the spatial aspect ratio of the Gabor function.

\subsection{Log Gabor Filters}

Gabor filters bandwidth is limited to one octave therefore it is not appropriate if we need broad spectral information. Log Gabor filters was proposed by Field in 1987. It can be constructed with arbitrary bandwidth. In Gabor filters is bandwidth is increased more than one octave, the DC component also increases, while Log Gabor filters by definition has no DC component. Log Gabor function have Gaussian transfer function viewed on logarithmic frequency scale, while Gabor function have Gaussian transfer function viewed on linear frequency scale. Log Gabor filters in the frequency domain can be represented by transfer function $H(f, \theta)$

$$
H(f, \theta)=H_{f} \times H_{\theta}
$$

$H_{f}$ is the radial component shown in Fig 4 controlling the bandwidth of the filter responds to and $H_{\theta}$ is the angular component as shown in Fig 5 controlling the spatial orientation of the filter. 2-D Log Gabor filters in polar form can be represented by the following equation:

$$
\begin{aligned}
H(f, \theta)=\exp & \left\{\left[\operatorname { l n } \left[\left(f / f_{0}\right]^{2}\right.\right.\right. \\
& / 2\left[\ln \left(\left(\sigma_{f} / f_{0}\right]^{2}\right\} \exp \{-(\theta\right. \\
& \left.\left.-\theta_{0}\right) / 2 \sigma_{\theta}^{2}\right\}
\end{aligned}
$$

where $f_{0}$ is the filter's center frequency is the filters direction. The constant $\sigma_{f}$ defines the radial bandwidth in octaves and the constant $\sigma_{\theta}$ defines the angular bandwidth in radian. To obtain constant shape ratio filters the term $\sigma_{f} / f_{0}$ must also be held constant for varying $f_{0}$. For example, a $\sigma_{f} / f_{0}$ value of .74 will result in a filter bandwidth of approximately one octave, .55 will result in two octaves, and .41 will produce three octaves.

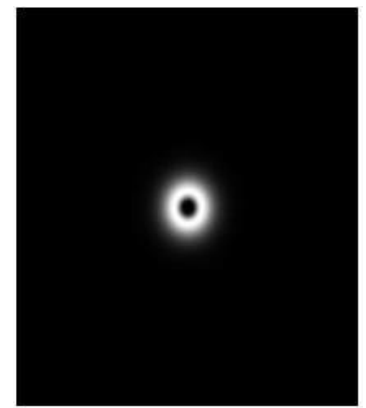

Fig 4: Radial component of Log Gabor filter

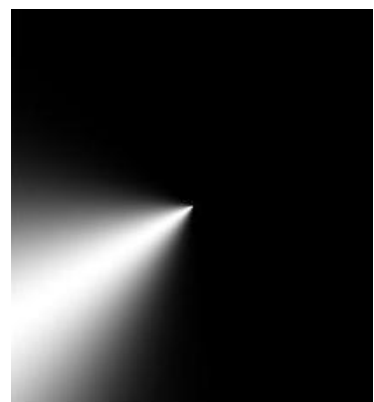

Fig 5: Angular component of Log Gabor filter 


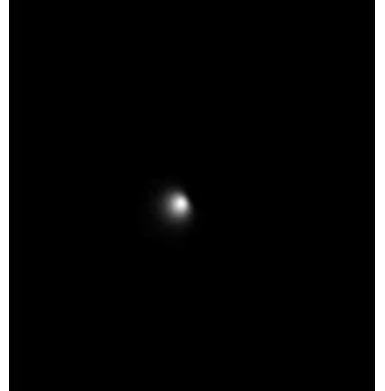

Fig 6: Product of radial and angular component of Log Gabor filter

\section{FEATURE REDUCTION}

The extracted features in the feature extraction phase are of very large dimension. It consists of some irrelevant information. The feature reduction process removes that irrelevant information by keeping important information that are necessary for recognition of expressions.

\subsection{Principal Component Analysis}

Principal Component Analysis (PCA) is a useful technique to reduce the dimensions of the dataset with minimal loss of information. Many of the pixels in feature vectors extracted during feature extraction process gives very little information for the classifier. These pixels can be removed using PCA. PCA is a linear transformation technique that transforms the data of possibly correlated variables to a set of values of linearly uncorrelated variables called principle components. In this transformation the first principal component is the one that has the largest possible variance, which shows the highest variability in the data as possible, and each succeeding component in turn has the next highest variance components. Low-order components often contain the most important aspects of the data so it is kept and high order components are removed. Steps to calculate PCA is given below.

1) All training set images are resized and converted in to a single vector.

2) The test image is resized and converted in to a single vector.

3) The mean image of all training set images plus test image is calculated.

4) Then the mean image is subtracted from each image of the training set as well as from the test image. After subtraction we will get new images called as difference images.

5) All difference images of training set as well as test image are converted in to a column vector i.e. column-wise concatenation of all images.

6) Then using covariance matrix the eigenvector and eigenvalues are calculated.

7) We have to choose the eigenvectors that we wish to keep in our data and formed a feature vector.

8) We take the transpose of the feature vector and multiply it on the left of the transpose of the mean centered data matrix to form the final data for both training and testing image.

\section{CLASSIFICATION}

In classification training images are trained and put into different classes of facial expressions. Then the test images are tested with the training images and their respective classes and output is found to which class the test image belongs to. Euclidean distance is used as a classifier in our work.

\subsection{Euclidean Distance}

The classifier based on Euclidean distance has been used which is obtained by calculating the distance between feature vectors of training and testing images. The Euclidean distance of a projected test image from each projected train image is calculated and the minimum value is chosen in order to find out the train image which is closest to the test image. The test image falls in the same class as that of the train image which is closest of all.

The formula for the Euclidean distance is given by:

$$
E D=\sqrt{\sum\left(x_{2}-x_{1}\right)^{2}}
$$

\section{RESULTS}

The database used is self-generated database. We evaluated the proposed algorithm by 42 still images. Three emotions (happy, neutral and surprise) are identified. Out of 42, 17 images are used in training and 25 as testing set. This algorithm also calculates the distance of test image from neutral image and the best match of test image from trained images.

Table 1. Recognition rate

\begin{tabular}{|c|c|c|c|c|c|}
\hline \multirow{2}{*}{ Expression } & \multicolumn{2}{|c|}{ Number of Images } & \multicolumn{3}{c|}{ Recognition rate (\%) } \\
\cline { 2 - 6 } & Training & Testing & PCA & Gabor & Log Gabor \\
\hline Happy & 7 & 9 & 44.4 & 77.7 & 100 \\
\hline Neutral & 7 & 9 & 66.6 & 77.7 & 88.8 \\
\hline Surprise & 3 & 7 & 57.1 & 71.4 & 71.4 \\
\hline \multicolumn{3}{|c|}{ Average } & 56.0 & 75.6 & 86.7 \\
\hline
\end{tabular}

\section{CONCLUSION}

In our self-generated database the accuracy rate of log Gabor filters $86.7 \%$ while that of Gabor filters is $75.6 \%$. Therefore $\log$ Gabor filters performs better than Gabor filters. When the face images are directly applied to PCA for feature reduction without passing through the feature extraction the accuracy rate is only $56 \%$ which is less than both Gabor and log Gabor filters. Frontal pose images can be detected and extracted correctly. Small amount of roll in the head position can be acceptable for the system. However large rotation of head or profile view is not acceptable. Low resolution images are not detected correctly.

\section{FUTURE SCOPE}

We have used static frontal face images in our experiment. Most of face image processing applications are video based. Therefore future work can be done on developing algorithms and code for real time analysis. Skin color modeling can be improved. Work can be done to extract broad skin color. Some improvements can be performed on recognition part. Firstly, number of people in the face database can be increased. Databases are very limited and are not easily available. Therefore number of database can be increased and that should be easily available for research work. Database 
should also contain subjects from different race, so that system can be developed which can detect face and expressions of person from different origin. Work can be done on detection of expression under various head position. Code can be developed to detect profile face.

\section{REFERENCES}

[1] Jyoti Mahajan, Rohini Mahajan, "FCA: A Proposed Method for an Automatic Facial Expression Recognition system using ANN", International Journal of Computer Applications (0975-8887), vol.-84, no.-4, 2013.

[2] Jeemoni Kalita, Karen Das, "Recognition of Facial Expression Using Eigenvector based Distributed Features and Euclidean Distance Based Decision making Technique", International Journal of Advance Computer Science and Applications, vol.-4, no.-2, 2013.

[3] Mahesh Kumbhar, Ashish Jadhav, and Manasi Patil, "Facial Expression Recognition Based on Image Feature", International Journal of Computer and Communication Engineering, vol.-1, no.-2, July 2012.

[4] Seyed Mehdi Lajevardi Hong Ren Wu, "Facial Expression Recognition in Perceptual color Space", IEEE Transaction on Image Processing, vol.-21, no.-8, pp. 3721-3732, August 2012

[5] D Murugan, Dr. S Arumugam, K Rajalakshmi, Manish $\mathrm{T}$, "performance evaluation of face recognition using Gabor filter, Log Gabor filter, and discrete wavelet transform", International journal of computer science \& technology(IJCSIT), vol.-2, no.-1, February 2010
[6] Saeid Fazli, Reza Afrouzian, Hadi Seyedarabi, “ High Performance facial Expression Recognition using Gabor Filter and Probabilistic Neural Network", 978-1-42444738-1/09, IEEE, 2009.

[7] Hong-Bo Deng, Lian-Wen Jin, Li-Xin Zhen, Jian- Cheng Huang, "A New Facial Expression Recognition Method Based on Local Gabor Filter Bank and PCA plus LDA", International Journal of Information Technology, vol.11, no.-11, 2005

[8] Maja Pantic, Michel Valstar, Ron Rademaker and Ludo Maat, "web-based database for facial expression analysis", 0-7803-9332-5/05, IEEE, 2005.

[9] A. Mehrabian, Nonverbal Communication. London, U.K.: Aldine, 2007

\section{AUTHOR'S PROFILE}

Shail Kumari Shah received the B.tech degree in Computer Science \& Engineering from RVS College of Engineering \& Technology in 2010 and she is pursuing her M.tech from RCEW, Jaipur. Her field of interest is Image Processing, data structure and database.

Vineet Khanna received his B.E and M.tech degree in Computer Science. He has work experience of 7.5 years. His area of interest is Computer Networks, Wireless Sensor Networks, Wireless Ad hoc Networks, Image Processing. He is now with RCEW, Jaipur Rajasthan, India. 